\title{
Éditorial
}

\section{Radiographies dentaires et méningiome ou quand le fanatisme fait oublier l'EBM !}

\author{
Marc El Hage, Jacky Samson* \\ «Les chiffres sont des êtres fragiles qui, à force d'être torturés, finissent par avouer tout ce \\ $q u$ 'on veut leur faire dire » Alfred Sauvy
}

Claus et al. ont publié en ligne sur le site du journal Cancer, le 10 avril 2012, un article sur « Les radiographies dentaires et le risque de méningiome » [1], dont les conclusions alarmistes ont été reprises par de nombreux média (par différents journaux comme L'Express, Le Monde, Le Figaro, Libération... en France, 20 Minutes, Le Temps... en Suisse ; par différentes télévisions comme RTL, TSR, BFMTV...). Ces différents média ignoraient l'article original mais ils ont reproduit scrupuleusement le communiqué de presse accompagnant la parution de cet article qui affirme que les radiographies dentaires augmenteraient le risque de survenue d'un méningiome. Le méningiome est une tumeur cérébrale fréquente, bénigne mais parfois agressive, qui présente la particularité de pouvoir être induite par les radiations.

Or, l'étude de Claus et al. montre seulement que des individus ayant eu un méningiome auraient eu plus d'examens radiographiques dentaires que le reste de la population [1]. Très rapidement, des experts ont réagi et critiqué la méthodologie de cette étude. Claus et al. ont contacté 1433 patients, âgés de 20 à 79 ans, ayant été traités pour un méningiome, mais ils n'ont pas consulté les dossiers médico-dentaires. La collecte des données a été effectuée grâce à un interrogatoire et un questionnaire (étude déclarative) : « Les participants ont été invités à préciser le nombre de fois qu'ils ont subi une radiographie interproximale (bite-wing), un status radiographique dentaire complet ou un cliché panoramique » au cours des différentes périodes de leur vie (avant l'âge de 10 ans, entre 10 et 19 ans, 20 et 49 ans et après 50 ans).

Cette étude repose donc sur des données approximatives : en l'absence de notes, qui se souvient exactement du nombre et du type de radiographies dentaires faites 10 ou 20 ans, voire plusieurs décennies auparavant ? De plus, ces données sont influencées par un phénomène bien connu, appelé « biais de rappel » ou «biais de mémorisation ». Celui-ci est observé quand des patients atteints d'une maladie identique (le méningiome dans cette étude) sont interrogés pour rechercher la cause éventuelle de leur maladie (les radiographies dentaires dans cette étude). Cette démarche a tendance à amener les patients à surévaluer le rôle de la cause suspectée (dans cette étude, le nombre de radiographies dentaires qu'ils ont effectuées). Ce biais a très probablement contribué à majorer largement le risque. Cette enquête montre que le risque de survenue d'un méningiome serait plus élevé de 1,2 à 2 fois pour les patients ayant subi une seule radiographie interproximale et de 1,0 à 1,2 fois pour les patients ayant eu un status radiographique dentaire complet (10 à 20 radiographies intrabuccales). Ces résultats sont de toute évidence incohérents car comment peut-on imaginer qu'une radiographie intra-buccale représente un risque plus élevé qu'une dizaine ou une vingtaine de radiographies intra-buccales ? L'incohérence des résultats enlève toute crédibilité à l'étude. En conséquence, on ne peut pas considérer qu'elle a démontré l'existence d'un lien entre les radiographies dentaires et la survenue d'un méningiome.

La publication de cette étude ou plutôt l'intervention des média ont suscité de vives réactions parmi les sociétés savantes intéressées qui toutes ont contesté en termes clairs la validité des résultats. L'American Academy of Oral and Maxillofacial Radiology (AAOMR) a conclu sa mise au point [5] de la façon suivante : «Il existe dans l'étude un certain nombre de problèmes de cohérence et de collecte des données qui constituent de graves lacunes et qui invalident les conclusions ». L'AAOMR

\footnotetext{
*Correspondance : jacky.samson@unige.ch
} 
faisait également remarquer que « Les doses absorbées (par les patients) lors des radiographies dentaires ont baissé d'environ $60 \%$ ces dernières années grâce à la commercialisation de nouveaux films radiologiques, au développement de la radiologie numérique, à une meilleure collimation du faisceau de rayons $X$ et à l'amélioration de la protection du patient ». On peut résumer cette intervention en deux mots : les résultats sont erronés et dépassés. Certes, les auteurs de l'étude ne sont pas spécialistes en radiologie mais on comprend mal leur démarche et on est surpris que l'étude similaire faite en 2004, publiée également dans la revue Cancer [6], et qui concluait qu'il n'y avait pas de risque de méningiome lié aux radiographies dentaires interpoximales, céphalométriques ou panoramiques, ne les ait pas davantage interpellés.

Il y a également un autre article [7] qui n'a pas interpellé les auteurs et qu'ils citent pourtant en référence. Dans cette étude, les auteurs ont cherché à identifier d'éventuels facteurs génétiques prédisposants à partir de nombreux cas de méningiomes radio-induits ou non, et en comparant les sujets irradiés qui ont développé un méningiome à ceux qui n'ont pas développé de méningiome. Les méningiomes radio-induits sélectionnés sont survenus chez des sujets qui ont eu une irradiation cérébrale pendant l'enfance pour traiter une teigne. De la fin du XIXème siècle jusqu'à l'apparition de la griséofulvine à la fin des années 50, la teigne a été traitée par radiothérapie, le plus souvent par un seul champ recevant 3,5-4 Gy ce qui correspond à une dose moyenne de 1,5 Gy pour le cerveau [2]. Sur plus de 20000 enfants irradiés en Israël entre 1949 et 1959, moins de $1 \%$ ont développé un méningiome [7].

Enfin, deux remarques pour clore le chapitre traitant de la légèreté scientifique des auteurs. Rappelons la définition du méningiome radio-induit: classiquement, le diagnostic ne peut être suspecté que s'il se développe dans le champ irradié, après une latence d'au moins 5 ans et que son aspect histologique est différent de celui de la tumeur initialement irradiée. Il constitue la tumeur cérébrale radio-induite la plus fréquente ( 5 fois plus fréquent que le gliome et le sarcome) et les lésions sont volontiers multiples, atypiques et récidivantes. Le méningiome radio-induit représente $1 \%$ des méningiomes. Voilà encore des éléments qui n'ont pas été pris en compte dans l'étude. À côté du méningiome iatrogène, Claus et al. ont rappelé l'existence de méningiomes chez les victimes de Hiroshima et Nagasaki en oubliant de préciser qu'il n'y a pas de risque statistiquement significatif de cancers pour des doses aux organes inférieures à 100 mSv, c'est-à-dire une dose correspondant à plus de 10000 radiographies panoramiques effectuées avec des appareils récents. La réalisation d'une radiographie panoramique expose à une dose de 4 à $30 \mu \mathrm{Sv}$; pour mémoire, la radioactivité naturelle (composée des irradiations d'origine tellurique, cosmique et alimentaire, et de celles dues au radon) est de 2,4 mSv par an, soit 80 fois la dose délivrée lors d'un examen effectué avec les appareils pour panoramique les plus exposants [8]. Plus concrètement, l'irradiation due à une radiographie intra-buccale correspond à un jour d'irradiation naturelle ou à celle délivrée par la consommation d'une à deux cigarettes. Quant à l'irradiation due à une radiographie panoramique, elle correspond, en fonction de l'appareil, à une irradiation naturelle d'un à cinq jours ou à celle délivrée par 1 à 10 cigarettes [4].

Par ailleurs, Claus et al. [1] affirment que les radiographies dentaires représentent la source d'irradiation artificielle la plus commune aux États-Unis; peut-être voulaient-ils dire celle dont les média parlent le plus ? Cette présentation spécieuse a été rapidement corrigée par exemple par l'Ordre des dentistes du Québec [3] - pays où la pratique radiologique est très proche de celle des Éats-Unis - : l'irradiation liée aux radiographies dentaires représente environ $3 \%$ de l'irradiation artificielle à laquelle la population générale est exposée, celle liée à la radiologie médicale $56 \%$ et celle liée à la médecine nucléaire $21 \%$; au total, l'irradiation artificielle représente $19 \%$ de l'irradiation totale, l'irradiation naturelle $81 \%$.

Suite à la polémique engendrée par la publication de cet article, l'auteur principal, Elizabeth Claus, a fait plusieurs déclarations pour répondre aux critiques et pour relativiser la portée de ses conclusions. Celle destinée aux patients constitue une véritable rétractation : «Ne paniquez pas, n'arrêtez pas de consulter votre dentiste, et consultez les recommandations de l'ADA (American Dental Association) afin d'en discuter avec votre dentiste pour voir s'il y aurait moyen de diminuer le nombre de radiographies ». Une nouvelle fois, on peut regretter que l'auteur principal ignore même les notions élémentaires de radiologie dentaire et le principe ALARA (As Low As Reasonably Achievable radiation exposure) appliqué par les chirurgiens dentistes.

En conclusion, il faut accorder peu de crédit à cette étude car l'abondance de statistiques ne peut compenser l'indigence des données épidémiologiques. Cette étude serait sans doute restée confidentielle si sa publication n'avait pas été accompagnée d'un communiqué de presse, procédé qui tend à se généraliser dans un monde scientifique où le faire savoir prend le pas sur le savoir-faire. Avec ce communiqué de presse, les plumitifs ont trouvé là une mauvaise nouvelle qu'ils se sont empressé de répandre et, comme le psittacisme est une véritable maladie endémique chez eux, aucun d'entre eux n'a cherché à prendre l'avis d'un expert avant de diffuser les données contenues dans ce communiqué de presse. Dans le monde scientifique, cette course effrénée à la notoriété ressemble à un sacrifice propitiatoire : pour la renommée du service, de l'institution, voire pour sa propre renommée, on a tendance à rechercher une mise en valeur immédiate et souvent un peu surfaite, d'autant plus facile à obtenir que les « autorités de surveillance » manquent curieusement de vigilance. En effet, on peut s'étonner que la revue Cancer, revue de l'American Cancer Society (impact factor 4,771 affiché sur le site) et les relecteurs acceptent la publication d'un article dont l'auteur principal est obligé par la suite de se rétracter publiquement 
et de s'adresser directement aux patients pour les rassurer. Cet auteur aurait peut-être dû prendre connaissance des recommandations de l'ADA avant d'entreprendre son étude et pas seulement les consulter pour pouvoir répondre aux critiques? Dans ce faux acte de contrition, l'auteur ne peut s'empêcher de revenir sur ce qui semblait bien constituer l'objectif premier de cette étude, amener les praticiens à diminuer le nombre de radiographies dentaires!

Conflits d'intérêt : aucun

\section{Références}

1. Claus EB, Calvocoressi L, Bondy ML, Schildkraut JM, Weimels JL, Wrensch M. Dental X-rays and risk of meningioma. Cancer 2012, doi: $10.1002 /$ cncr.26625.

2. Cribier B. Teignes et teigneux. Radiotherapy and tinea capitis a 100 years ago. Ann Dermatol Venereol 2011;138:553-5.
3. Forest D. Radiographie dentaire. Mise au point de l'ODQ. Complément d'information. www.odq.qc.ca

4. Foucart J-M. La radioprotection en odontologie. Collection Mémento. CdP, Vélizy, 2004.

5. Lam E, Yang J. AAOMR response to recent study on dental X-ray risks. www.aaomr.org

6. Longstreth WT Jr, Phillips LE, Drangsholt M, Koepsell TD, Custer BS, Gehrels J-A, van Belle G. Dental X-rays and the risk of intracranial meningioma. Cancer 2004;100:1026-34.

7. Sadetzki S, Flint-Richter P, Starinsky S, Novikov I, Lerman Y, Goldman B, Friedman E. Genotyping of patients with sporadic and radiation-associated meningiomas. Cancer Epidemiol Biomarkers Prev 2005;14:969-76.

8. Unité cancer et environnement. Note d'information sur l'étude Claus, 2012. Dental X-rays and risk of meningioma. 2012. www.cancer-environnement.fr 